\title{
Supramolecular chemical shift reagents inducing conformational transitions: NMR analysis of carbohydrate homooligomer mixtures
}

Beeren, Sophie; Meier, Sebastian

Published in:

Chemical Communications

Link to article, DOI:

10.1039/C4CC09710F

Publication date:

2015

Document Version

Peer reviewed version

Link back to DTU Orbit

Citation (APA):

Beeren, S., \& Meier, S. (2015). Supramolecular chemical shift reagents inducing conformational transitions:

NMR analysis of carbohydrate homooligomer mixtures. Chemical Communications, 51, 3073-3076.

https://doi.org/10.1039/C4CC09710F

\section{General rights}

Copyright and moral rights for the publications made accessible in the public portal are retained by the authors and/or other copyright owners and it is a condition of accessing publications that users recognise and abide by the legal requirements associated with these rights.

- Users may download and print one copy of any publication from the public portal for the purpose of private study or research.

- You may not further distribute the material or use it for any profit-making activity or commercial gain

- You may freely distribute the URL identifying the publication in the public portal

If you believe that this document breaches copyright please contact us providing details, and we will remove access to the work immediately and investigate your claim. 


\section{Supramolecular chemical shift reagents inducing conformational transitions: NMR analysis of carbohydrate homooligomer mixtures}

Received 00th January 2012 Accepted 00th January 2012

DOI: $10.1039 / \times 0 \times x 00000 x$

\author{
Sophie R. Beeren*a and Sebastian Meier*b
}

www.rsc.org/

\begin{abstract}
We introduce the concept of supramolecular chemical shift reagents as a tool to improve signal resolution for the NMR analysis of homooligomers. Non-covalent interactions with the shift reagent can constrain otherwise flexible analytes inducing a conformational transition that results in signal separation. Here we use this approach for the quantitative analysis of a complex homooligomeric glycan mixture.
\end{abstract}

The analysis of complex mixtures is routinely achieved via the physical separation of components on the basis of differential noncovalent interactions with a stationary phase. ${ }^{1}$ Chromatographic and electrophoretic methods can exploit specific interaction with a chosen ligand ${ }^{2,3}$ or utilise the differential partitioning of analytes with a micellar pseudo-stationary phase. ${ }^{4,5} \mathrm{NMR}$ spectroscopic analysis of mixtures relies upon differences between the chemical structures of the analytes to achieve chemical shift resolution and therefore the analysis of mixtures of chemically similar analytes, for example homooligomers, is a challenge. ${ }^{6}$ In this study, we show that the addition of guests introducing conformational transitions in the analytes is a powerful method to achieve signal dispersion in NMR spectroscopy, whereby the guest functions as a type of noncovalently bound affinity-based diamagnetic chemical shift reagent. Here, therefore," non-covalent interactions are exploited to obtain a spectroscopic separation of mixture components rather than a physical separation, which enables the analysis of complex mixtures in situ.

Many carbohydrate polymers, for example cellulose, chitin and starch, are homopolymers formed via identical glycosidic bonds, which makes the analysis of biological and biotechnological processes involving these materials somewhat challenging. Chromatographic and electrophoretic methods to analyse oligosaccharides can be time-consuming and often require prederivatization with an optically active label. Ordinarily NMR spectroscopy is of very limited use for the analysis of homooligosaccharides. For $\alpha(1-4)$ linked glucans, the signals for different length oligomers are completely overlapped in 1-D ${ }^{1} \mathrm{H}-\mathrm{NMR}$ spectra (ESI Figure S2(a)) and using high resolution $2 \mathrm{D}{ }^{1} \mathrm{H}^{13} \mathrm{C}$ experiments only mixtures with degree of polymerisation (DP) ranging from glucose (G1) to maltohexaose (G6) can be resolved even with an $800 \mathrm{MHz}$ spectrometer. ${ }^{6 a}$

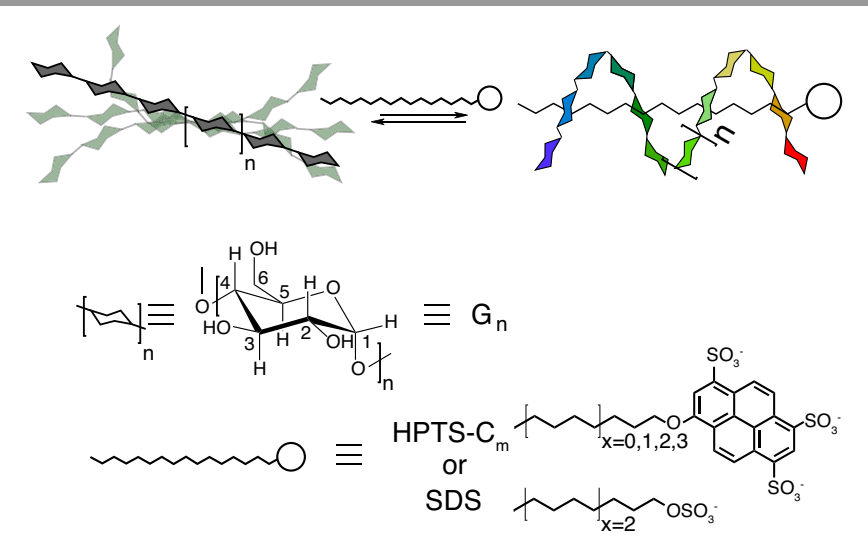

Fig. 1 . Schematic representation of how mixtures of short homooligomeric $\alpha(1-4)$ glucans exist in aqueous solution as unstructured populations with highly overlapped NMR spectra but complexation with suitable amphiphilic shift reagents results in a conformational transition, whereby anomeric (H1) signals can be resolved using ${ }^{1} \mathrm{H}-{ }^{13} \mathrm{C}$ HSQC spectra.

Improved NMR analysis of complex carbohydrate mixtures may be feasible using complexation with suitable probes.' It has been widely recognized that conformationally flexible biomolecules can gain structural definition upon complexation with a suitable interaction partner in a coupled binding and folding reaction, leading to vastly improved chemical shift dispersion. ${ }^{8}$ Herein we describe how the resolution and quantification of homooligomeric $\alpha$-glucans with $\mathrm{DP}<14$ can be achieved using NMR spectroscopy by exploiting the conformational constraints resulting from the interaction of $\alpha(1-4)$-glucans with the hydrophobic aliphatic tail of surfactant-like amphiphiles (Figure 1).

Recently, we have characterized the binding behaviour of amphiphiles to $\alpha(1-4)$-glucans by monitoring the ${ }^{1} \mathrm{H}$ chemical shift changes of the amphiphilic probe. ${ }^{9}$ The observed chemical shift changes and intermolecular nOes upon binding were consistent with the binding of the guest to the hydrophobic interior of a singlestranded left-handed helix (Figure 1). This mode of solution-state binding mimics that reported for the solid state encapsulation of 
lipids and surfactants by $\alpha$-glucans studied using crystallography ${ }^{10}$ and solid-state ${ }^{13} \mathrm{C}$ NMR spectroscopy ${ }^{11}$ and is furthermore supported by $\alpha$-glucan binding studies in solution using CD spectroscopy, ${ }^{12}$ as well as molecular modelling. ${ }^{13}$ We further demonstrated using this system how binding constants for the interaction of a guest with multiple different hosts can be determined simultaneously from a single NMR titration so long as signals from the various resulting host/guest complexes can be resolved. ${ }^{14}$ Herein we show that a sizedependent conformational transition in the carbohydrate hosts resulting from an interaction with tailor-made amphiphiles leads to the resolution of signals from different length $\alpha(1-4)$ glucans in NMR spectra and we evaluate the benefits of these supramolecular shift reagents for the quantitative analysis of complex homooligomeric mixtures in situ. Contributions of the headgroup and tails of different length in the supramolecular shift reagents are systematically analysed using commercial and rationally designed guests.

${ }^{1} \mathrm{H}-{ }^{13} \mathrm{C}$ HSQC experiments were used to examine the influence of amphiphile complexation on a mixture of $\alpha(1-4)$ glucans with natural ${ }^{13} \mathrm{C}$ isotope abundance and DP 1-20 (see ESI Figure S1 for length distribution). The use of ${ }^{1} \mathrm{H}^{13} \mathrm{C}$ HSQC is beneficial due to (1) to the absence of homonuclear couplings in the ${ }^{13} \mathrm{C}$ dimension at natural isotopic abundance, (2) the large ${ }^{13} \mathrm{C}$ chemical shift range, (3) usually long $T_{2}$ relaxation times and narrow ${ }^{13} \mathrm{C}$ line widths in oligosaccharides and (4) the sensitivity benefits of indirect ${ }^{13} \mathrm{C}$ detection in an experiment using ${ }^{1} \mathrm{H}$ excitation and detection. ${ }^{15}$ Figure $2 \mathrm{~b} / \mathrm{c}$ shows the effect of adding $10 \mathrm{mM}$ sodium dodecylsulfate (SDS) to the $\alpha(1-4)$-glucan mixture $(10 \mathrm{mg} / \mathrm{ml})$ in $\mathrm{D}_{2} \mathrm{O}$ on the anomeric $\mathrm{H} 1$ signals of the oligosaccharides. In carbohydrates, conformational freedom largely resides in the glycosidic bond and thus chemical shift changes upon conformational transitions of oligosaccharides especially affect the atom groups in the glycosidic bonds (in this case $\mathrm{H} 1$ and $\mathrm{H} 4$ ). Considering the presence of

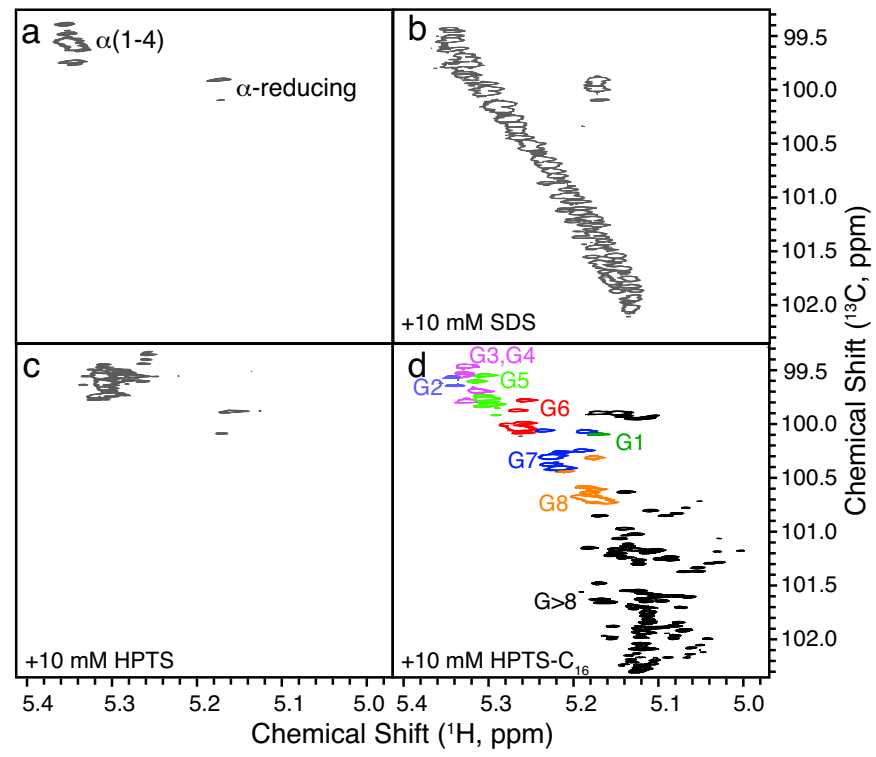

Fig. $2 \cdot{ }^{1} \mathrm{H}-{ }^{13} \mathrm{C}$ HSQC spectral regions $\left(\mathrm{D}_{2} \mathrm{O}, 800 \mathrm{MHz}, 300 \mathrm{~K}\right)$ showing the anomeric H1-C1 groups in (a) a mixture of $\alpha(1-4)$ glucans $(10 \mathrm{mg} / \mathrm{ml})$ and the same mixture in the presence of (b) 10 mM SDS, (c) 10 mM HPTS, and (d) $10 \mathrm{mM}$ HPTS-C 16 . oligomers with DP 1-13 at detectable concentrations and the presence of $\alpha$-and $\beta$-anomeric reducing ends, 26 molecules with 182 distinct anomeric $\mathrm{CH}$ groups occur in the mixture. While ${ }^{1} \mathrm{H}-{ }^{13} \mathrm{C}$ HSQC spectra are highly congested in pure $\mathrm{D}_{2} \mathrm{O}$ (Figure 2a), the addition of SDS fans out the $\alpha$-anomeric signals along a diagonal in the ${ }^{1} \mathrm{H}-{ }^{13} \mathrm{C}$ $\mathrm{HSQC}$ spectrum resolving as many as 130 anomeric signals, yielding a high-resolution spectral map of chemically highly similar analytes. Specifically, the presence of SDS for inducing conformational transitions and concomitant chemical shift changes results in a resolution improvement of at least 6-fold in both spectral dimensions. Conventional NMR spectroscopy in the absence of the shift reagent would thus require an at least 6-fold higher magnetic field to reach the same signal resolution. In the presence of SDS, a nearly perfect linear correlation between ${ }^{1} \mathrm{H}$ and ${ }^{13} \mathrm{C}$ chemical shifts is observed (Figure $2 \mathrm{~b}$ ). The linear relationship suggests that the chemical shifts result from a weighted average of the rapid two-state exchange between an unbound population of flexible oligomers and a bound population in which the oligomers are conformationally constrained, where the bound population is greater for longer carbohydrates that bind with higher affinities.

While complexation with SDS results in signal separation in the anomeric spectral region, the assignment of anomeric signals to specific residues of identified oligosaccharides was not possible. The influence of binding to a tailor-made highly water soluble amphiphilic probe HPTS- $\mathrm{C}_{16}$ (HPTS = 8-hydroxypyrene-1,3,6trisulfonic acid trisodium salt), utilised in previous studies ${ }^{9}$ was therefore investigated. Figure $2 \mathrm{~d}$ illustrates the additional dispersion of $\mathrm{H} 1$ signals in the ${ }^{1} \mathrm{H}$ dimension resulting from the incorporation of an aromatic head group onto the amphiphile, enabling the resolution of $\sim 160$ distinct anomeric signals. HPTS- $\mathrm{C}_{16}$ has a dual effect upon binding to oligosaccharides: (1) it causes a conformational transition to the oligosaccharide upon helix formation resulting in a downfield shift in the ${ }^{13} \mathrm{C}$ dimension and upfield shift in the ${ }^{1} \mathrm{H}$ dimension, and (2) it introduces a ring current effect resulting from the aromatic head group, which gives an additional upfield shift in the ${ }^{1} \mathrm{H}$ dimension. For comparison, the spectrum of the same oligosaccharide mixture is shown in the presence of the HPTS head group alone, wherein no signal dispersion is seen (Figure 2c); HPTS itself does not bind to oligosaccharides and the ring current of the HPTS head group is only felt when the amphiphile's tail is present to cause a strong enough binding interaction. Signals from the $\alpha(1-4)$-glucan mixture in the presence of HPTS- $\mathrm{C}_{16}$ were assigned by comparison to complexes of the pure reference compounds for the readily commercially available maltooligosaccharides up to DP 8 . The non-linearity of the spectral map in the presence of HPTS- $C_{16}$ suggests an increasing influence of helix formation upon increasing length of the oligosaccharide, but an aromatic ring current effect that is most pronounced at approximately DP 10 . This finding can be explained by an increase in the binding strength ${ }^{14}$ and helix definition upon increasing oligosaccharide length $\left(K_{\mathrm{a}}(\mathrm{DP} 5)=370 \mathrm{M}^{-1}\right.$ as compared to $K_{\mathrm{a}}(\mathrm{DP} 12)=$ $\left.15000 \mathrm{M}^{-1}\right)$, concomitant with a reduced average influence of the ring current effect on $\mathrm{H} 1$ signals, as there are increasingly more $\mathrm{H} 1$ protons situated at a greater distance from the aromatic head group.

Compared with chromatographic methods for mixture analysis, the presence of several signals per molecular species in NMR spectra 
may appear to complicate the analysis of mixtures using NMR spectroscopy. However, this level of resolution provides chemical detail for instance for studying binding modes in supramolecular complexes by observing the chemical shift changes of individual sites. While the $\alpha$-anomeric region of the ${ }^{1} \mathrm{H}-{ }^{13} \mathrm{C} \mathrm{HSQC}$ spectra shown in Figure 2 is highly complex we noticed that the $\mathrm{H} 1, \mathrm{H} 2$ and $\mathrm{H} 5$ signals of the $\beta$-reducing end glucose unit of different length oligosaccharides in a mixture exhibited particularly well-defined and well-dispersed signals in the presence of HPTS- $C_{16}$ (ESI Figure S3). Figures $3 a, b$ and $f$ display the three characteristic spectral regions containing signals from for the glucan mixture in the presence of 10 $\mathrm{mM}$ HPTS- $\mathrm{C}_{16}$. We suggest that the dispersion in the ${ }^{1} \mathrm{H}$ dimension results primarily from a ring current effect and influences these protons and especially $H 1 \beta$ and $H 5 \beta$ due to their proximity to the aromatic head group: $\mathrm{H} 1 \beta, \mathrm{H} 3, \mathrm{H} 5$ and $\mathrm{H} 6$ point towards the centre of the helix when an oligosaccharide wraps around a hydrophobic guest. ${ }^{9}$ The importance of amphiphile tail length for the observation of this phenomenon was investigated by varying the length from $C_{16}$ to $C_{12}, C_{8}$ and $C_{4}$ (Figure 3c-f and ESI Figure S4). While the $C_{12}$ amphiphile was as effective as $C_{16}$ at dispersing the $\beta$-reducing end oligosaccharide signals, the amphiphile with a $\mathrm{C}_{4}$ aliphatic tail did not induce any significant signal dispersion possibly due to its reduced hydrophobicity and therefore poor binding affinity. Meanwhile the $C_{8}$ amphiphile could disperse the signals up to a maximum oligosaccharide length of DP 10, whereafter signals for longer $\alpha$ glucans were overlapped. We expect that this signal overlap is due to the fact that 10 glucose units are sufficient to wrap around an alkane with 8 carbon units and each additional glucose unit therefore has a minimal effect on the strength of the binding interaction and on the proximity of reducing end and the HPTS moiety. A similar length limit of resolution most likely exists for the $C_{12}$ and $C_{16}$ amphiphiles but this limit occurs at DP $>13$. The observed upper limit of DP 13 is a consequence of the low concentration of longer glucans in the mixture and thus a limitation by sensitivity rather than resolution. Signal resolution increases with increasing concentration of the added amphiphile relative to the analyte concentration and $10 \mathrm{mM}$ was found to give sufficiently good signal dispersion for this 10 $\mathrm{mg} / \mathrm{ml}$ oligosaccharide sample. Accordingly, lower-field NMR instrumentation (below $800 \mathrm{MHz}$ ) would be expected to suffice for resolving the mixture up to $d p 13$ in the case of sufficiently sensitive NMR instrumentation and additional amphiphile could be added as required.
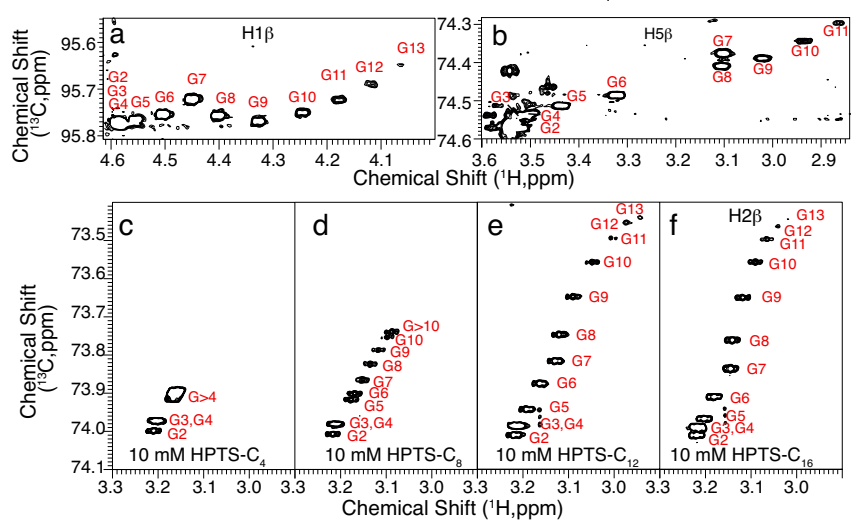

Fig. $3{ }^{1} \mathrm{H}-13 \mathrm{C}$ HSQC spectral regions $\left(\mathrm{D}_{2} \mathrm{O}, 800 \mathrm{MHz}, 300 \mathrm{~K}\right)$ showing (a) H1 and (b) $\mathrm{H} 5$ signals of the reducing end glucopyranose of the $\beta$-anomer for a mixture of $\alpha$-glucans $(10 \mathrm{mg} / \mathrm{ml})$ in the presence of HPTS- $C_{16}(10 \mathrm{mM})$ in $D_{2} \mathrm{O}$ and (c-f) the $\beta$-reducing end $\mathrm{H} 2$ signal in the presence of $10 \mathrm{mM} \mathrm{HPTS}^{-} \mathrm{C}_{4}$ (c), HPTS-C $_{8}(d)$, HPTS-C$_{12}$ (e) and HPTS-C 16 (f).

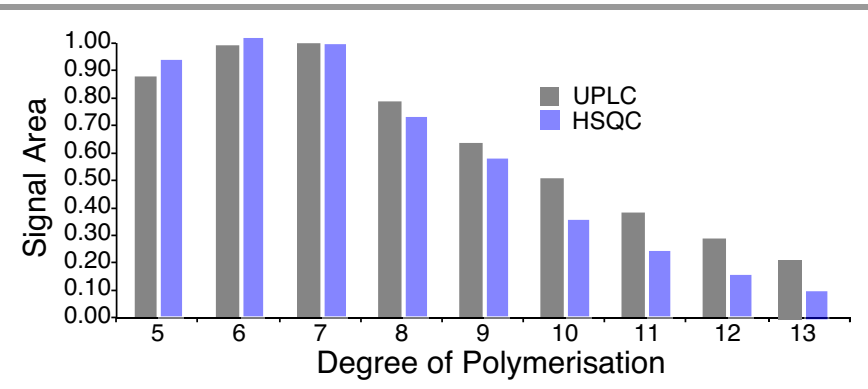

Fig. 4 Fluorescence signal areas for the $\alpha$-glucan mixture analysed with UPLC after prederivatization (grey) and ${ }^{1} \mathrm{H}-13 \mathrm{C}$ HSQC signal areas for the reducing end ${ }^{1} \mathrm{H} 1-{ }^{13} \mathrm{C} 1$ of the $\beta$-anomers in the underivatized mixture (light blue).

Through the enhanced resolution of oligosaccharide signals in HSQC provided by the addition of our amphiphilic shift reagent, in situ quantification of oligosaccharide concentrations was made possible. Signals from the $\beta$-reducing end were integrated and the relative concentrations in the mixture were compared to chromatographic quantifications obtained using UPLC on the analyte mixture pre-derivatised with a 2-aminobenzamide fluorescent label at the reducing end (Figure 4 and ESI Figure S1). UPLC quantifications were directly compared to HSQC signal volumes for signals with $\mathrm{DP}<14$. Even in the absence of correction for the different relaxation behaviour of the HSQC signals from $\alpha(1-4)$-glucans with different length, a convincing agreement was found between HSQC and UPLC determinations. The underestimation of HSQC signal areas for longer $\alpha(1-4)$-glucans is consistent with increasing relaxation losses for the longer analytes. The agreement could be further improved by the use of standard curves for quantification or by HSQC experiments that account for the differential loss of magnetization during polarization transfer pathways. ${ }^{16}$

In conclusion, we have described how the binding of tailor-made amphiphiles by mixtures of $\alpha(1-4)$ glucans imposes structural definition on the otherwise conformationally flexible oligosaccharides resulting in a length-dependent chemical shift change. As a consequence of the increased resolution of signals in ${ }^{1} \mathrm{H}-{ }^{13} \mathrm{C}$ HSQC spectra, the quantitative in situ analysis of a complex $\alpha(1-4)$ glucan mixture is made possible using NMR spectroscopy. Surfactants have previously been used in matrix-assisted diffusion ordered spectroscopy (DOSY) to affect the diffusion properties of mixture compounds and resolve them through micellar partitioning. ${ }^{6 f}$ Here we have shown that surfactants can also be directly used for their specific interaction as complexing agents to distinguish different analyte sizes through differential chemical shift changes resultant upon binding induced conformational transitions. We propose that a range of carefully selected supramolecular shift reagents could play a role in the NMR-based mixture analysis of homooligomers generally, and work towards this goal is on-going.

NMR spectra were recorded on the $800 \mathrm{MHz}$ spectrometer of the Danish National Instrument Center for NMR Spectroscopy of Biological Macromolecules at the Carlsberg Laboratory and the 
Technical University of Denmark. S.M. acknowledges funding by the Carlsberg Foundation grant 2013_01_0709.

\section{Notes and references}

${ }^{a}$ Carlsberg Laboratory, Gamle Carlsberg Vej 10, Copenhagen V 1799 , Denmark. Email: sophie.beeren@carlsberglab.dk

${ }^{b}$ Technical University of Denmark, Department of Chemistry, Kemitorvet, Building 201, Kgs. Lyngby, 2800, Denmark. Email: semei@kemi.dtu.dk

Electronic Supplementary Information (ESI) available: detailed description of NMR experiments and additional relevant spectra. See DOI: $10.1039 / \mathrm{c} 000000 \mathrm{x} /$

1 (a) C. E. Weill and P. Hanke, Anal. Chem., 1962, 34, 1736; (b) P. Jandera, Anal. Chim. Acta, 2011, 692, 1.

2 P. Cuatrecasas and C. B. Anfinsen, in Methods in Enzymology, ed. B. J. William, Academic Press, 1971, pp. 345-378.

3 T. C. Bøg-Hansen, Anal. Biochem., 1973, 56, 480.

4 A. L. Shapiro, E. Viñuela and J. V. Maizel Jr, Biochem. Biophys. Res. Commun., 1967, 28, 815.

5 S. Terabe, K. Otsuka, K. Ichikawa, A. Tsuchiya and T. Ando, Anal. Chem., 1984, 56, 111.

6 (a) M. Bojstrup, B. O. Petersen, S. R. Beeren, O. Hindsgaul and S. Meier, Anal. Chem., 2013, 85, 8802; (b) S. Huang, J. Gao, R. Wu, S. $\mathrm{Li}$ and Z. Bai, Angew. Chem. Int. Ed., 2014, 53, 11592; (c) K. F. Morris, P. Stilbs and C. S. Johnson, Anal. Chem., 1994, 66, 211; (d) Z. Pan and D. Raftery, Anal. Bioanal. Chem., 2007, 387, 525; (e) A. K. Rogerson, J. A. Aguilar, M. Nilsson and G. A. Morris, Chem. Commun., 2011, 47, 7063; (f) C. F. Tormena, R. Evans, S. Haiber, M. Nilsson and G. A. Morris, Magn. Res. Chem., 2010, 48, 550.

7 J. F. Teichert, D. Mazunin and J. W. Bode, Journal of the American Chemical Society, 2013, 135, 11314.

8 P. E. Wright and H. J. Dyson, Curr. Opin. Structural Biol., 2009, 19, 31.

9 (a) S. R. Beeren and O. Hindsgaul, Angew. Chem., 2013, 52, 11265; (b) S. R. Beeren, S. Meier and O. Hindsgaul, Chem. Eur. J, 2013, 19, 16314.

10 K. Gessler, I. Usón, T. Takaha, N. Krauss, S. M. Smith, S. Okada, G. M. Sheldrick and W. Saenger, Proc. Nat. Acad. Sci., 1999, 96, 4246.

11 (a) M. J. Gidley and S. M. Bociek, J. Am. Chem. Soc, 1988, 110, 3820; (b) C. E. Snape, W. R. Morrison, M. M. Maroto-Valer, J. Karkalas and R. A. Pethrick, Carbohydr. Polym., 1998, 36, 225.

12 (a) B. Pfannemüller, H. Mayerhöfer and R. C. Schulz, Biopolymers, 1971, 10, 243; (b) G. Wulff, S. Kubik, Carbohydr. Res. 1992, 237, 1.

13 C. A. López, A. H. de Vries and S. J. Marrink, Carbohydr. Res., 2012, 364, 1 .

14 S. Meier and S. R. Beeren, J. Am. Chem. Soc., 2014, 136, 11284.

15 B. O. Petersen, O. Hindsgaul and S. Meier, Analyst, 2014, 139, 401.

16 (a) K. Hu, W. M. Westler and J. L. Markley, J. Am. Chem. Soc., 2011, 133, 1662; (b) I. A. Lewis, S. C. Schommer, B. Hodis, K. A. Robb, M. Tonelli, W. M. Westler, M. R. Sussman and J. L. Markley, Anal. Chem., 2007, 79, 9385 